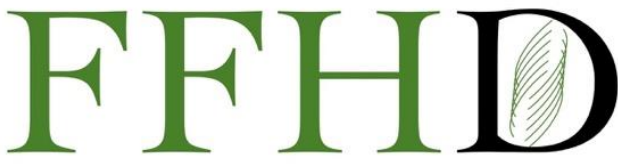

Functional Foods in Health and Disease

\title{
Fasting and fasting mimetic supplementation address sirtuin expression, miRNA and microbiota composition
}

\section{Stephanie Lilja ${ }^{1}$, Angelika Pointner ${ }^{1}$, Hanna Bäck ${ }^{1}$, Kalina Duszka ${ }^{1}$, Berit Hippe ${ }^{1}$, Lucia Suarez ${ }^{1}$, Ingrid Höfinger ${ }^{2}$, Tewodros Debebe ${ }^{3}$, Jürgen König ${ }^{1}$, Alexander G. Haslberger ${ }^{1}$}

${ }^{1}$ Department of Nutritional Sciences, University of Vienna, 1090 Vienna, Austria, ${ }^{2}$ Monastory, Pernegg, ${ }^{3}$ Biomes NGS GmbH, Germany

*Corresponding author: Alexander Haslberger, Ph,D., Department of Nutritional Sciences, University of Vienna, 1090 Vienna, Austria

Submission Date: September 28 ${ }^{\text {th }}, 2020$; Acceptance Date: October $22^{\text {nd }}, 2020$; Publication Date: October 30.2020

Please cite this article as: Lilja S., Pointner A., Bäck H., Duszka K., Hippe B., Suarez L., Höfinger I., Debebe T., König J., Haslberger A. Fasting and fasting mimetic supplementation address sirtuin expression, miRNA and microbiota composition. Functional Foods in Health and Disease 2020; 10(10): 439-455. DOI: https://www.doi.org/10.31989/ffhd.v10i10.752

\footnotetext{
Abstract

Background: Fasting and fasting mimetics - bioactive compounds mimicking fasting effects, are of growing interest as potential means to slow down the aging process and increase health span. Sirtuins are known as enzymes that interfere with mitochondrial energy metabolism and molecular pathways involved in Periodic fasting vs. fasting mimicking supplementation

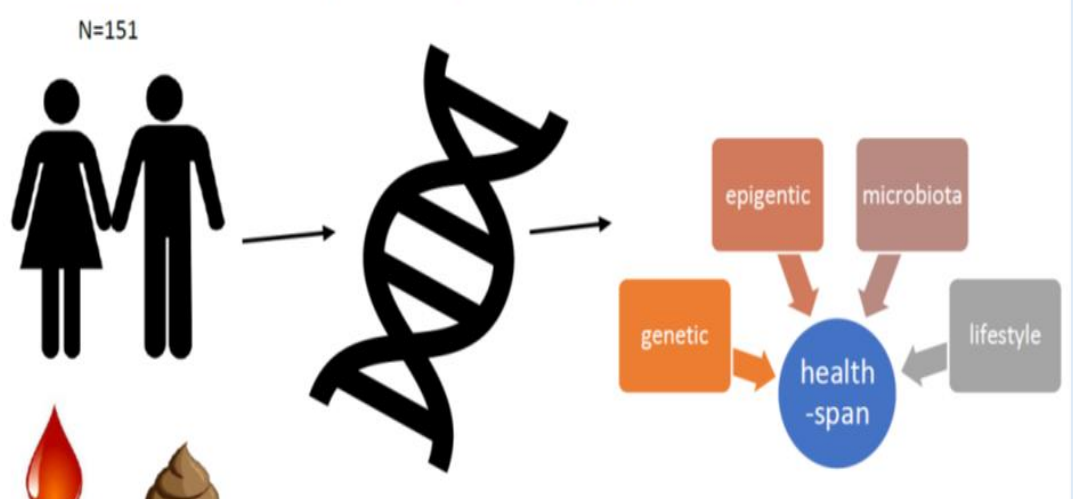
longevity. Although their activation is determined as a response to stress i.e. caloric restriction. Sirtuin activating nutraceuticals are believed to mimic the effects of nutrient deprivation, thus activating signaling pathways correlated to an improved health span. In this study, we compare 5 days periodic buchinger fasting intervention with 3 months shot supplementation, a drink formula, containing secondary plant ingredients considered to activate sirtuins.
} 
Methods We analyzed pathways in response to fasting and a sirtuins activating drink. Genetic and epigenetic biomarkers including telomere length, LINE1 methylation, and a set of mRNAs and miRNAs were assessed using qPCR analysis. Gut composition and metabolites were compared using Illumnia sequencing and mass spectrometry.

Results Fasting, but also the fasting mimetic could increase expression of FoxO1, SIRT1, and MLH1 mRNA, all genes discussed in aspects of longevity. A positive correlation between telomere length and both SIRT1, and SIRT6 was observed. Furthermore, a significant change in the gut composition was measured. Actinobacteria increased in the supplementation group, whereas after buchinger fasting a rise in the distribution of Proteobacteria could be observed. Firmicutes/Bacteroidetes ratio decreased and correlated with the body mass index (BMI).

Conclusions Our results confirm the effects of fasting on longevity associated mechanisms but also suggest that SIRTFOOD shot intervention addresses some of these effects.

(CFFC 2020. This is an Open Access article distributed under the terms of the Creative Commons Attribution 4.0 License (http://creativecommons.org/licenses/by/4.0)

\section{INTRODUCTION}

The hallmarks of aging are often summarized as genomic instability, epigenetic modification, telomere shortening, epigenetic alterations, altered intracellular communication and more[1]. Epigenetic mechanisms are agreed to be at the centre of regulation of aging and the epigenetic clock is considered to be the most reliable marker for aging. As age increases, reactive oxygen species (ROS) amass and drive senescence pathways, impairing epigenetic methylation of CPGs, histones as well as miRNAs. Aging can address various mechanisms differently and recently, even certain age types were defined ${ }^{2,3}$. Calorie restriction (CR) has been discussed as a potential method of delaying the onset of aging conditions associated with increased morbidity and mortality. CR is defined as a moderate reduction of around $20-40 \%$ calorie intake compared to ad libitum without malnutrition and was found to slow down aging and extend healthspan[4,5]. Periodic fasting, like 'buchinger fasting' with a total daily intake of $<250 \mathrm{kcal}$ is mostly unstudied.

During fasting pyruvate dehydrogenase kinase isoform 4 (PDK4) is upregulated, muscle and liver stores of glycogen are depleted to release glucose into the bloodstream ${ }^{6,7}$. Then, ß-oxidation provides energy by metabolizing the released free fatty acids to ketone bodies to serve as an alternative energy source[8,7]. The energy status in the cell is reflected by the adenosine monophosphate (AMP)/ adenosine triphosphate (ATP) ratio, which first increases during fasting or $\mathrm{CR}$ and triggers $\mathrm{AMP}$-activated protein kinase (AMPK). AMPK promotes an intracellular increase in nicotinamide adenine dinucleotide (NAD $)$ levels, the rate-limiting substrate for silent information regulator 2 (SIR2), which is one of the critical mediators of CR-induced lifespan extension in yeast. In mammals, 7 sirtuins, SIRT1-7, have been 
identified[10,9]. Sirtuins are located in different parts of the cells and have multiple functions including DNA repair, cell survival, interaction with metabolism, lipid and glucose homeostasis, stress resistance as well as insulin secretion[11,12]. SIRT1 is the most studied isoform among family members. It is associated with longevity and is often observed to be expressed in calorie-restricted cells[12]. A secondary molecular link between AMPK, CR, and SIRTs provides the Forkhead box subgroup O (FoxO) family[9]. The different transcription factors of this family promote fatty acid oxidation, suppress the generation of ROS and are linked to autophagic processes, thus slowing the accumulation of oxidative damage that might accelerate aging. FoxO is activated by its phosphorylation via AMPK and its activity is controlled through acetylation and deacetylation, which is altered by SIRT1. This further illustrates the networking interaction between AMPK, FoxO and SIRT1 in the regulation of many cellular processes involved in the adaptations to $\mathrm{CR}$ and promotion of longevity[9,13]. However, CR can affect gene expression through multiple mechanisms, like chromatin modifications, mRNA transcription and mRNA translation. In particular, control of the expression of regulatory RNAs such as microRNAs (miRNAs) is an important determinant in this regard[15]. MiRNAs have a variety of important functions including their modulating role in cell proliferation, differentiation, apoptosis and senescence[16]. mRNA can have several miRNAs regulators and vice versa ${ }^{17}$. More than 16 miRNAs regulate SIRT1 expression and activity under which
miR34a has been the most studied. MiRNAs do not only have a crucial impact on a human's physiology, they are also able to enter microbial organisms. The host's miRNA may potentially affect the gut microbial ecosystem. The composition of the intestinal bacterial community can influence the digestion of various dietary compounds and thus, affect the host's health. Food components can themselves also impact the growth and metabolic activity of gut microbiota, their composition and/or potential functions[18,19]. These include secondary plant ingredients or other bioactive substances, known as functional food or nutraceuticals - a dietary supplement that in addition to its nutritional value, beneficially modulates body functions[19].

Recently, the terms CR mimetics or fasting mimetics have been used for substances that mimic the biological effects of nutrient deprivation involving the activation of different signaling pathways with beneficial effects in metabolism, DNA repair and thus improving health span[20,21,22]. Polyphenols show increasing evidence to enhance the function of immune cells i.e natural killer cells to eliminate senescent cells. Moreover, multiple bioactive compounds, like EGCG, resveratrol, phloretin, butyrate and many more can act as epigenetic modifiers, modulate gene expression, DNA methylation and miRNAs[21,23]. Based on the observation that fasting and certain bioactive compounds have strongly overlapping physiological features regarding their impact on age-related pathways, this study has been conducted to compare the outcomes of buchinger fasting with a dietary supplementation containing different secondary plant ingredients, considered to activate sirtuins, and 
their relevance for salutogenesis ${ }^{24} .151$ healthy men and women were included. We measured and compared the relative telomere length, Long Interspersed Nuclear Elements (LINE1) methylation, expression of 6 different mRNAs and 7 different miRNAs as well as the changes in the gut composition.

\section{Material and Method}

Experimental design: 180 people were enrolled for the study performed at the University of Vienna, Department for nutritional science. The study included three different intervention groups: buchinger fasting, fasting mimetics supplementation and placebo/control. After dropouts the fasting intervention group consisted of 20 people, the 131 that attended the fasting mimetic supplementary intervention, were divided into 100 participants for the active supplement and 31 participants consuming placebos, acting as the control group. The subjects were between 21 and 75 years (mean 43 years). The mean BMI was $26.255 \mathrm{~kg} / \mathrm{m} 2 \pm 4.545 \mathrm{~kg} / \mathrm{m} 2$ and weight $76.562 \mathrm{~kg} \pm 15.420 \mathrm{~kg} .44$ participants (29.1\%) were male and 107 participants (70.9\%) were female. 124 participants of the total study population were nonsmokers and two thirds indicated to be physical active at least once a week. Exclusion criteria were metabolic diseases thus its medication. Moreover, participants taking probiotics, antibiotics or sirtuin activating compounds/medicine like metformin were excluded.

Dietary intervention: 20 participants joined a oneweek fasting program prescribed by Dr. Buchinger in Pernegg Monastery, defined and supervised by Mrs. Ingrid Höfinger, a fasting coach. The fasting program is described elsewhere[25] but in brief, consists of a total caloric intake of 250 calories a day for 5 consecutive days. The supplementation group was divided into a placebo control group and an active group. The supplement was composed by the Swiss company System-Biologie AG (Wollerau) and will be addressed as SIRTFOOD shot. Both groups were advised to not change their lifestyle and nutritional habits during the intervention time. Participants had to include one flacon $(25 \mathrm{ml})$ of SIRTFOOD shot or placebo during the day for three consecutive months. The study was randomized, controlled and singleblinded. The supplement consists of prebiotic and secondary plant ingredients, known to act as fasting mimetics and able to active sirtuins according to literature. The following compounds can be found in one falcon $(25 \mathrm{ml})$ SIRTFOOD shot: $3.5 \mathrm{mg}$ of gallic acid, $40 \mathrm{mg}$ EGCG, $25 \mathrm{mg}$ phloretin, $14 \mathrm{mg}$ anthocyanin, $10 \mathrm{mg}$ anthocyanidins, $6.4 \mathrm{mg}$ oleuropein and $0.9 \mathrm{mg}$ sulforaphane, $3 \mathrm{~g}$ galactooligosaccharides. The compounds were extracted from mango, apple, blueberry, olive leaves, broccoli and green tea.

Sample collection: Food frequency questionnaires, dried blood spots, and stool samples were collected at two different timepoints at the beginning and after the intervention periods. After collection, stool samples were stored at $-80^{\circ} \mathrm{C}$. In accordance with the declaration of the Viennese Human Ethics committee all study participants gave written consent for the use of data. The beginning of the intervention is defined as timepoint 1 (T1). For fasting, the end of the intervention was defined as timepoint 2 (T2) and for the SIRTFOOD shot intervention as timepoint 3 (T3).

LINE1 methylation and relative telomere length: Total DNA and RNA were isolated from dried blood spots (DBS) using MagMAX FFPE DNA/RNA ultra-kit (Thermofisher, USA) via KingFisher Duo Prime 
purification system. Quantity and quality of isolated RNA were assessed using a Nanodrop ND-1000 spectrophotometer (Nanodrop, Wilmington, DE, USA). For analysis of LINE1 methylation, qPCR and high-resolution melt analysis were applied. EpiTect HRM PCR kit (Qiagen) and primers were used as described below, and analysis was carried out in a Rotor Gene Q (Qiagen). Unknown study samples were compared to standards of known methylation, generated with REPLI-g Mini Kit and mixed accordingly, to generate standards with $0,25,50,75$ and $100 \%$ methylation. Bisulfit conversion was done using the EpiTect bisulfite kit (Qiagen) following the manufacturers' protocol and using a maximum of $2 \mu \mathrm{g}$ of genomic DNA. Relative telomere length was determined in genomic DNA isolated from the dried blood spots using a StepOne Plus real time PCR Detection System (Applied Biosysteme). For PCR, single-copy gene primers, telomere primers (Biomers, Germany) and a LightCycler ${ }^{\circledR} 480$ Sybr $^{\circledR}$ Green I master mix (Roche) were used. Relative telomere length was calculated using the formula of $2-\Delta \Delta \mathrm{Ct} \quad(\Delta \mathrm{Ct}=$ $\left.\mathrm{Ct}^{\text {Telomere }}-\mathrm{Ct}^{\text {Reference }} ; \Delta \Delta \mathrm{Ct}=\Delta \mathrm{Ct}-\Delta \mathrm{Ct}^{\text {mean placebo controls }}\right)$ as described elsewhere ${ }^{26}$. Primers for analysis of LINE1 and telomere length were selected from literature and purchased from Biomers, Germany. The primers were following: LINE1 forward TGTTAGATAGTGGGTGTAGGTT; for the reverse primer a 1:1 mixture was used from following primers LINE1 reverse 1: AATACATCCGTCACCCCTTT, LINE1 reverse 2: AAATACATCCATCACCCCTTT. Telomere forward CGGTTTGTTTGGGTTTGGGTTTGGGTTTGGGTTTGGGTT; telomere reverse GGCTTGCCTTACCCTTACCCTTACCCTTACCCTTACCCT telomere standard TTAGGGTTAGGGTTAGGGTTAGGGTTAGGGTTAGGGTTAGGGTTAGG GTTAGGGTTAGGGTTAGGGTTAGGGTTAGGGTTAGG, single copy gene forward CAGCAAGTGGGAAGGTGTAATCC, single copy gen reverse CCCATTCTATCATCAACGGGTACAA, single copy gene standard CAGCAAGTGGGAAGGTGTAATCCGTCTCCACAGACAAGGCCA GGACTCGTTTGTACCCGTTGATGATAGAATGGG. Each sample was done in duplicates.

miRNA and mRNA expression: Changes in miRNA and mRNA expression for genes associated with antiaging and longevity (FoxO1, SIRT1, PDK4, MLH1) were determined using commercially available primers (Thermofisher, USA). cDNA of mRNA was done using LunaScript RT SuperMix Kit (Biolabs, Germany). cDNA synthesizing from miRNA was done using TagMan Advanced miRNA cDNA synthesis kit and reverse transcription was conducted using a MultiGene gradient Thermal Cycler (Labconsulting). Samples were run in $10 \mu \mathrm{L}$ reactions in doubles, using TaqMan Fast advanced Mastermix (Thermofisher, USA) for RTqPCR amplifications performed on StepOne Plus realtime PCR Detection System (Applied Biosystem). All target mRNAs were normalized to GAPDH as an endogenous control. For target miRNAs, miR24 was used as an endogenous control. Fold changes for mRNA and miRNA were calculated using the $\Delta \Delta$ cycle threshold $\left(\Delta \Delta \mathrm{C}_{T}\right)$ method, with fold changes expressed relative to the mean values for the control group, placebo using the formula as described for the telomere length[27].

Microbiota and microbial metabolites: Upon sample collection, stool samples were stored at $-80^{\circ} \mathrm{C}$. For sequencing microbial composition all fasting samples were analyzed by Biomes NGS GmbH (Germany) using Illumina Sequencing. Microbial metabolites were analyzed using mass spectrometry at University of Vienna. 
SCFA MCFA analysis: The detection technique was established based on the published method 2-NPH or 3-NPH derivatised fatty acids analysis utilising LC$\mathrm{MS}^{28}$. Shortly dried pellets were resuspended in $150 \mu \mathrm{l}$ acetonitrile, samples were centrifuged at 14000rpm at $4^{\circ} \mathrm{C}$, dried in the SpeedVac concentrator The metabolites were analysed by liquid chromatography coupled to mass spectrometry (LC-MS) using an Ultimate 3000 (Thermo Fischer Scientific, Waltham, Massachusetts, US) and a micrOTOF-Q II (Bruker Daltonics, Bremen, Germany) with an Atlantis T3 $3 \mu \mathrm{m}$ column (2.1x150mm, Waters, Milford, MA, USA) kept at $40^{\circ} \mathrm{C}$.

Beta-hydroxybutyrate: BHB was measured from the fasting group only via on call GK Dual blood glucose and ketone meter (swiss point of care) using blood drops from finger before and after intervention.
Statistical analysis: All data are presented as mean \pm standard deviation (SD). Data were analyzed using IBM SPSS Statistics for Windows Version 22.0 (IBM Corp., Armonk, NY, USA) and graph pad prism (Version 6). Paired t-test was used to compare the different timepoints for parametric and Wilcoxon test for nonparametric values.

\section{RESULTS}

BHB and weight management: Fasting intervention resulted in ketogenesis with ß-hydroxybutyrate (BHB) increasing significantly after 5 days of fasting $(p<0.01)$ (Figure 1A). The participants lost on average $4.5 \mathrm{~kg}$, whereas participants in the supplementation groups lost $0.5 \mathrm{~kg}$ on average (Figure 1B). Ketone bodies were not measured in participants of the SIRTFOOD shot intervention, because ketogenesis is not expected when continuing dietary habits with normal caloric intake.
A

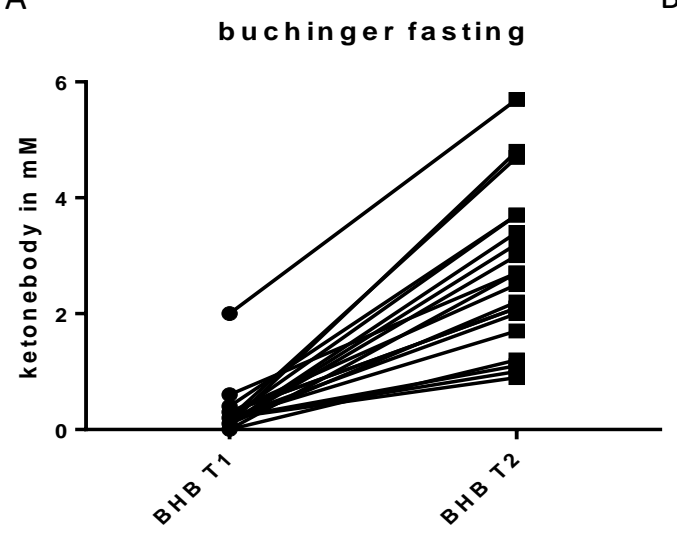

B

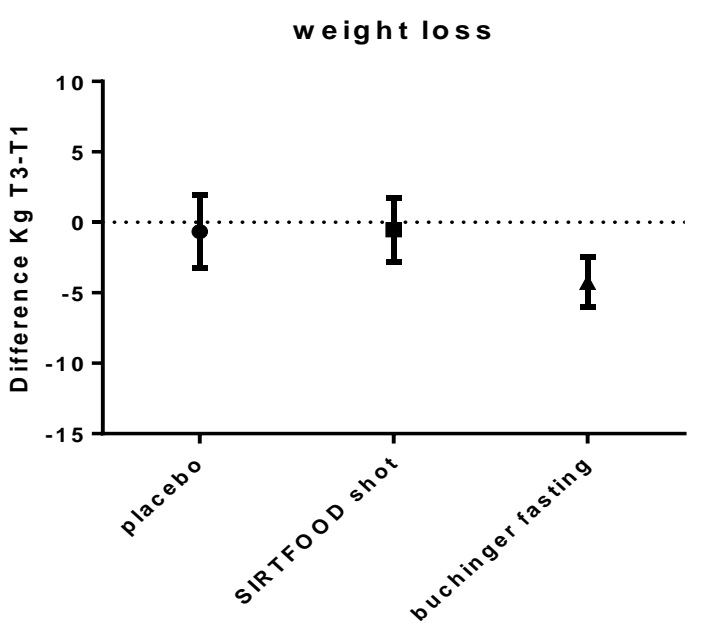

Figure 1: Ketogenesis measured by ketonebody BHB before and after buchinger fasting $(A)(p<0.01)$. Weight loss with different interventions pictured in (B). 

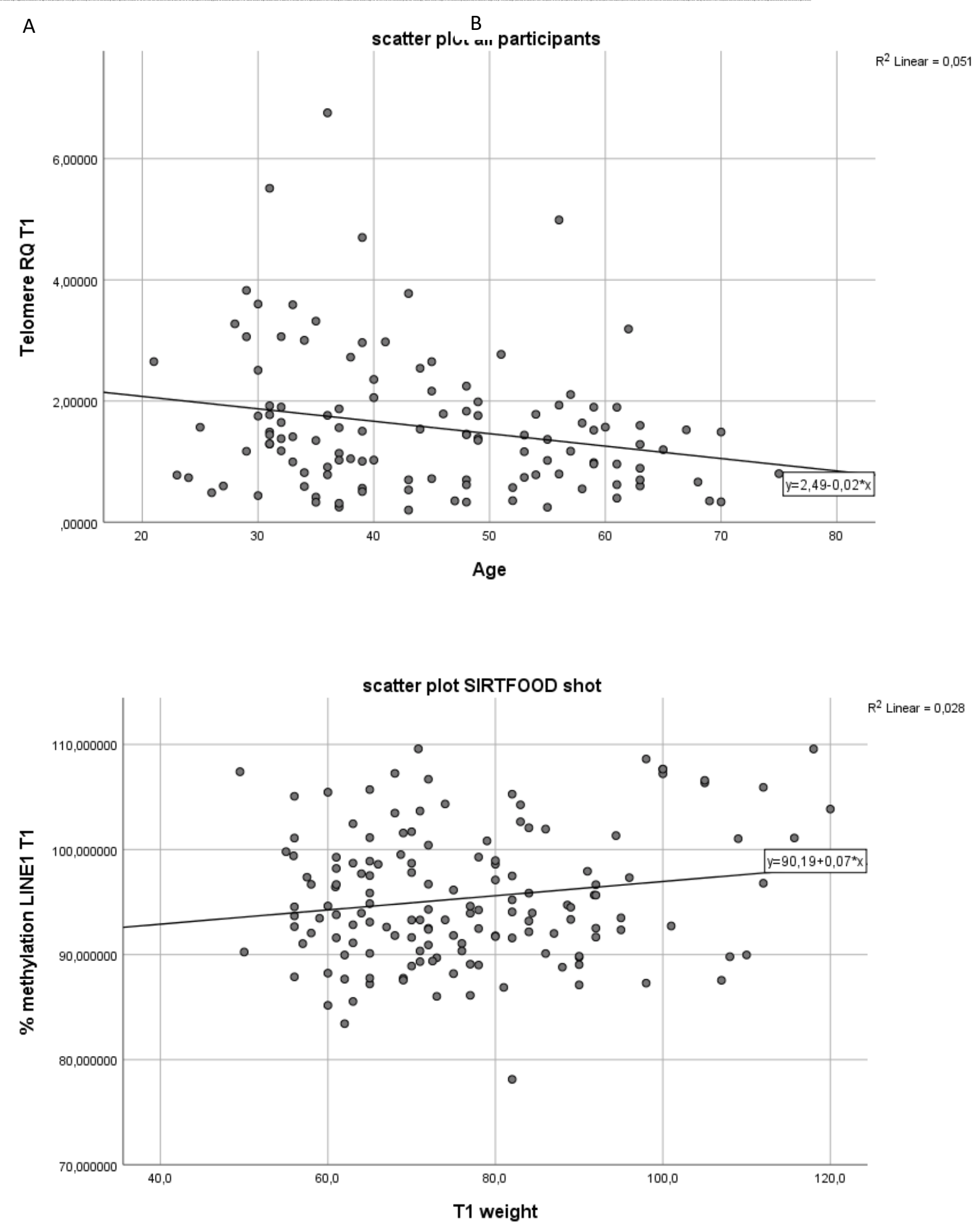

Figure 2: Output scatter plot spss, Pearson's correlation. Negative correlation of relative telomere length and age of all participants $(p<0.05)(A)$. LINE1 methylation positively correlated with weight before starting the SIRTFOOD shot intervention $(p<0.05)(B)$. 
A buchinger fasting

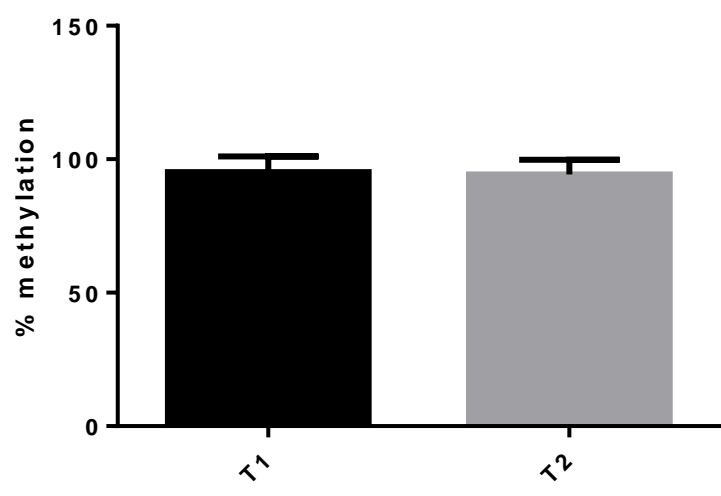

LINE1
B

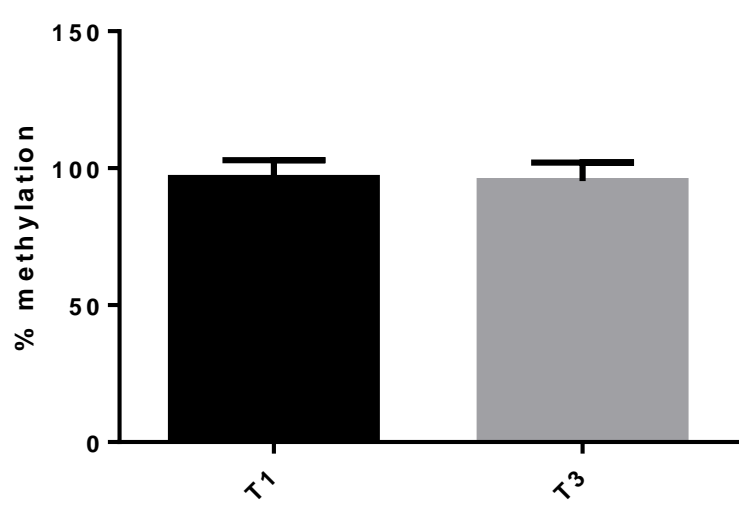

LINE 1

Figure 3: \% LINE1 methylation for buchinger fasting and SIRTFOOD shot. No differences could be seen before and after the interventions in LINE1 methylation (A\&B).

\section{Expression of mRNA}

Both buchinger periodic fasting and SIRTFOOD shot intervention could increase mRNA gene expression relevant for mechanisms involved in longevity pathways. Both interventions significantly increased FoxO1, MLH1, PDK4 and SIRT1 gene expression (Figure 4A \&B). SIRT3 was elevated in the fasting group, but not significantly and SIRT6 showed no effect (Figure 4B). SIRTFOOD shot had no effect on SIRT3 gene expression, whereas mRNA levels of SIRT6 decreased but not significantly (Figure 4A).

\section{Expression of miRNAs}

A set of different miRNAs was chosen depending on having an impact on the selected mRNAs or relevant for health span and longevity pathways. In the SIRTFOOD shot intervention significant changes were seen for miR93-5p, miR16-5p, miR21-5p and miR34a5p. MiR125b-5p decreased and miRlet $7 b-5 p$ elevated but not in a significant extend (Figure $5 \mathrm{~A}$ ). buchinger fasting ameliorated miR125b-5p, miR93-5p, miR16$5 p$, miR21-5p and attenuated miR34a-5p significantly. No significant effect was seen for miRlet $7 b-5 p$ (Figure $5 B)$. No changes in miR155- $5 p$ were seen in all samples. MiR122-5p and miR33b-5p were under the detective rate, therefore, these two miRNAs were excluded.

\section{Correlations MRNA and miRNA}

A significant correlation was observed between the different mRNA or miRNA gene expressions. For the fasting group, FoxO1 positively correlated with SIRT1, SIRT3 and MLH1 $(p<0.05)$ and MLH1 positively correlated with all genes besides SIRT6 $(p<0.01)$. Moreover, B-oxidation is controlled by PDK4, which overexpression increased SIRT1 and SIRT3 expression $(p<0.02)$. Telomere length and SIRT1 expression positively correlated at the beginning of the fasting intervention $(p<0.02)$ (Figure $8 A)$, but not for SIRTFOOD shot. Nevertheless, for the SIRTFOOD shot intervention multiple correlations were observed as well, like FoxO1 upregulation positively increased MLH1, PDK4 and SIRT1 gene expression. Connections between miRNA and mRNA expression were also seen. SIRT1 and miR34a-5p showed a negative correlation in the SIRTFOOD shot intervention, although only a strong trend. Telomere length positively correlated with SIRT6 and miR125b-5p expression for the supplementation intervention $(P<$ 0.05 ) at the baseline. 

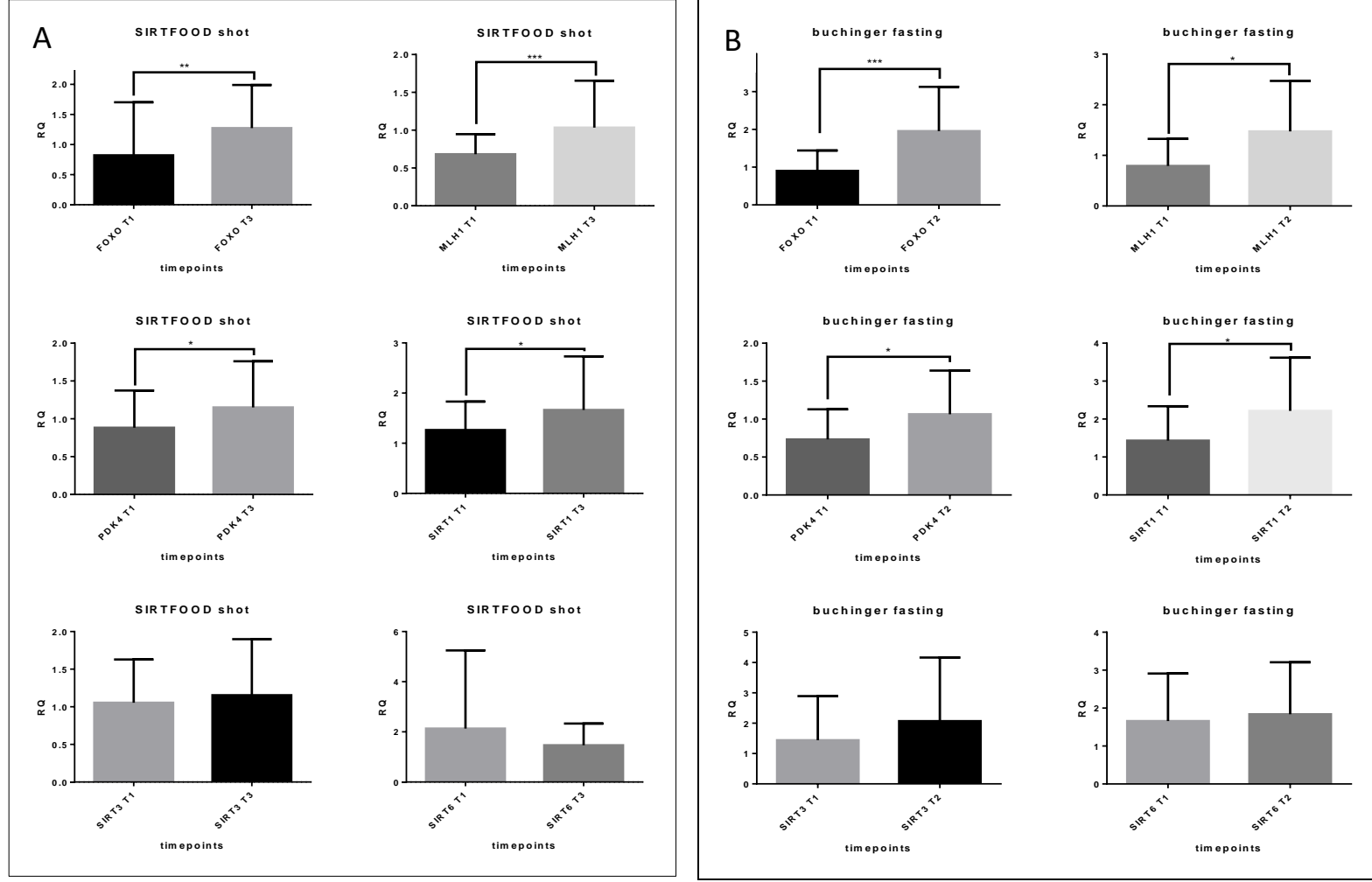

Figure 4: RQ selected mRNA gene expression (FoxO1, MLH1, PDK4, SIRT1, SIRT3, SIRT6) SIRTFOOD shot and buchinger fasting. The results are expressed as mean +/- SD. Statistical significance between timepoint 1 (T1) and end (T2 or T3) of the intervention was determined using paired t-test for parametric values and Wilcoxon test for nonparametric values.
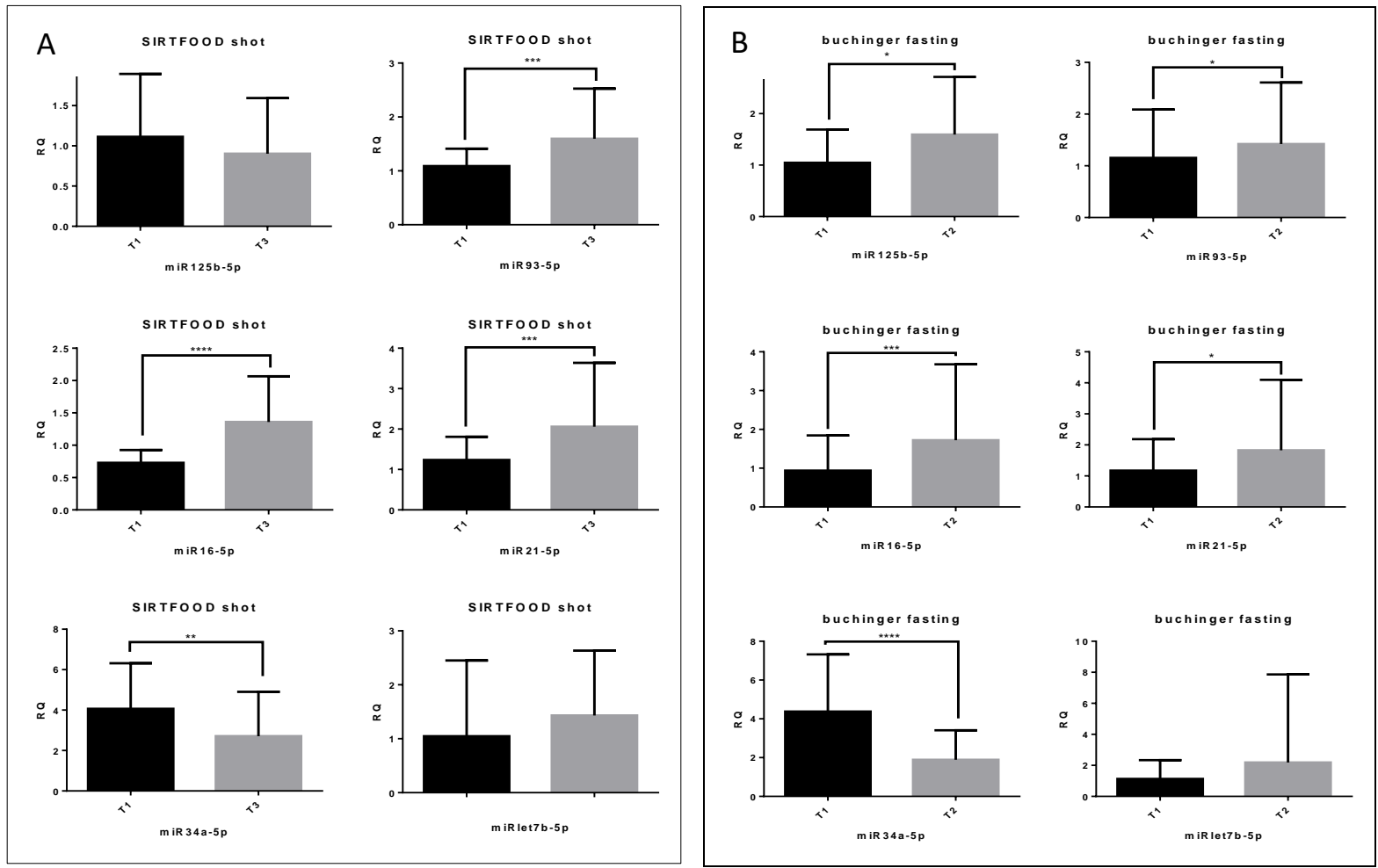

Figure 5: RQ selected miRNA gene expression (miR125b-5p, miR93-5p, miR16-5p, miR21-5p, miR34a-5p, miRlet7b-5p) SIRTFOOD shot and buchinger fasting. The results are expressed as mean +/- SD. Statistical significance between timepoint 1 (T1) and end (T2 or T3) of the intervention was determined using paired t-test for parametric values and Wilcoxon test for nonparametric values. 


\section{Microbiota and metabolites}

No changes for Actinobacteria, Bacteroidetes and Firmicutes were seen after the fasting intervention. However, Lentisphaerae decreased, Tenericutes and Verrucomicrobia elevated, but not significantly. A strong trend was seen in the switch of the abundance of Euryarchaeota and Cyanobacteria. Latter was increased after the intervention, whereas Euryarchaeota attenuated after periodic fasting. Significant elevated changes in microbiota were observed for Proteobacteria, TM7 and Fusobacteria (all $p<0.05$ ) (Figure 6A). For the SIRTFOOD shot intervention, a different pattern in the switch of microbiota can be seen. Tenericutes had the same result comparing buchinger fasting and SIRTFOOD shot intervention. Three months of supplementation lead to an increase in Euryarchaeta, Bacteroidetes, Lentisphaerae and decreased Cyanobacteria and Verrcoumicrobia but not in a significant manner. In comparison to periodic fasting where a significant increase can be seen for Proteobacteria, TM7 and Fusobacteria, SIRTFOOD shot decreased the amount of these phyla but not significantly. Nevertheless, a strong trend in Firmicutes reduction and a significant elevation in Actinobacteria ( $p<0.05)$ was observed with the supplement (Figure 6B). For the placebo group, no changes were seen in any of the phyla (Figure 6B). On the level of short-chain fatty acids (SCFAs) produced by bacteria, no changes can be seen after the SIRTFOOD shot intervention, whereas periodic fasting significantly increased butyrate level (Figure 7A). At the end of the intervention, a positive correlation can be observed for abundance in Tenericutes and the amount of butyrate produced $(p=$ 0.05). Although only a strong trend, medium-chain fatty acid (MSCFa) caprylate decreased after periodic fasting, but not for the other intervention. Statistical interactions with miRNAs and phlya were seen for Cyanobacteria and miR16-5p, with a positive correlation after the fasting intervention. The same phylum and additionally Lentisphaerae positively correlated with miR34a-p5 at the beginning of fasting $(p<0.02)$. Interestingly, SIRT1 expression positively correlated with the abundance of Actinobacteria and negatively with Cyanobacteria at the end of fasting, although not significantly. Nevertheless, SIRT1 expression positively correlated with Fusobacteria after an intervention ( $p<$ 0.05). In the supplementation group, Bacteroidetes positively affected miR125b-5p (Figure 8B), miR16-5p, miRlet7b-5p, MLH1 ( $p<0.05)$ and SIRT1 expression, although only a trend. Moreover, strong trends were seen for Actinobacteria and Cyanobacteria with different miRNAs, such as Verrucomicrobia and miR34a-5p. Latter bacterial phylum also significantly correlated with SIRT6 expression in a positive extend but decreases with age, observed for the fasting group $(p<0.05)$ Significant interactions are seen for Firmicutes/Bacteroidetes ratio with BMI and weight for all the participants and the SIRTFOOD shot intervention $(p<0.05)$ (Figure $8 C \& D)$.
A

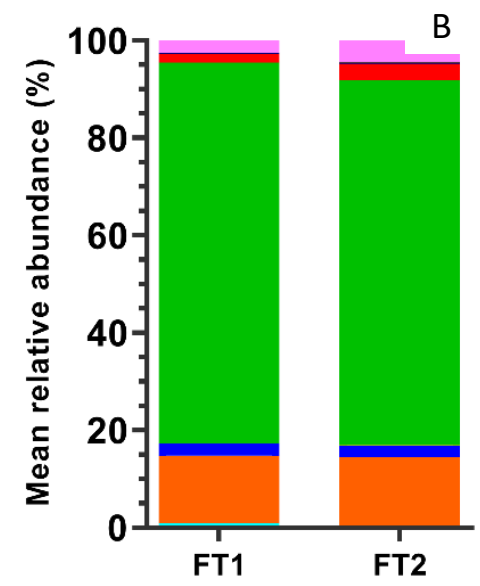

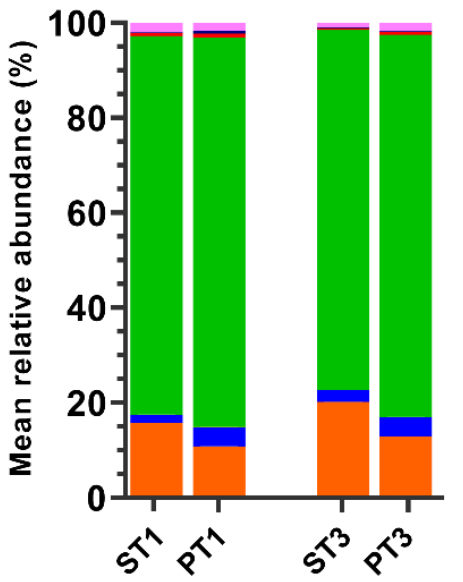

Others

Fusobacteria

Lentisphaerae

Verrucomicrobia

TM7

Tenericutes

Proteobacteria

Firmicutes

Cyanobacteria

Bacteroidetes

Actinobacteria

Euryarchaeota

Figure 6: Abundance microbiota by phyla for fasting group (A), SIRTFOOD shot (ST1 vs ST3) (B) and placebo group (PT1 vs PT3) (B). Results are expressed in percentage of the mean of relative abundance for the different phyla. Statistical significance between timepoint 1 (T1) and end (T2 or T3) of the intervention was determined using paired t-test for parametric values and Wilcoxon test for nonparametric values. 


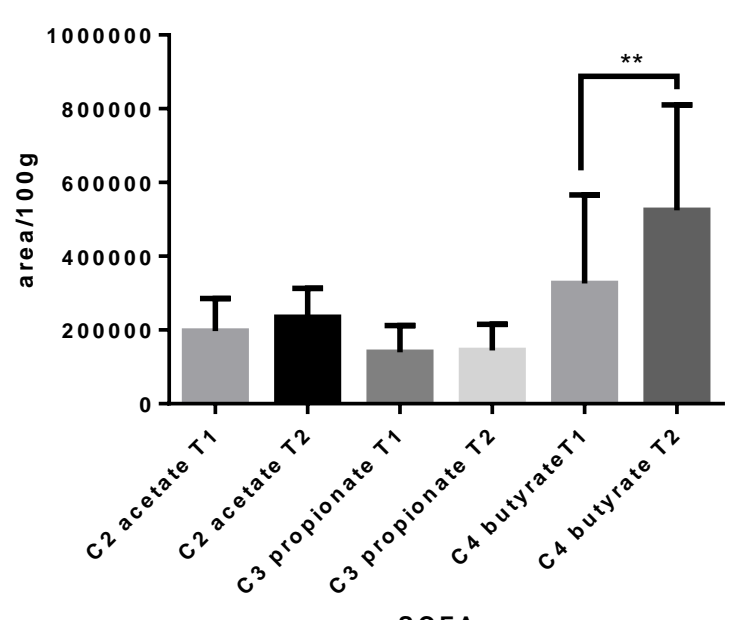

SIRTFOOD shot

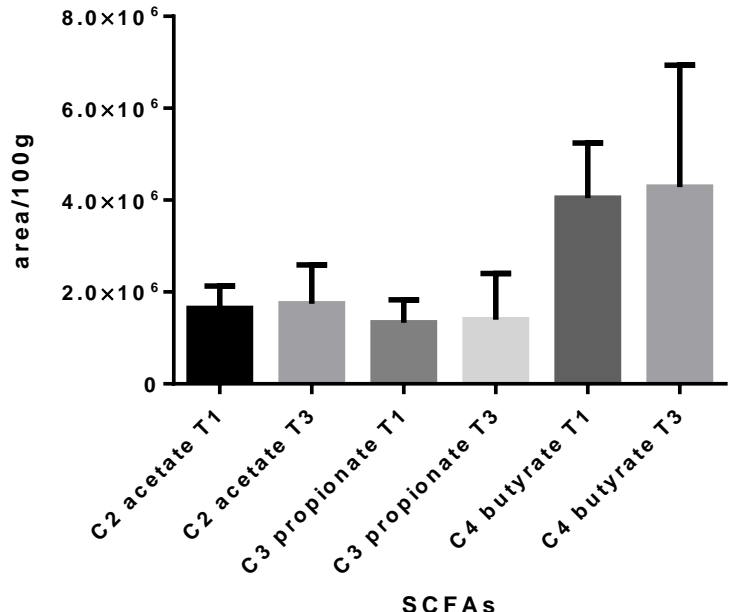

Figure 7: Amount of SCFAs produced given as area/100g stool for buchinger fasting (A) and SIRTFOOD shot (B) interventions. Statistical significance between timepoint 1 (T1) and end (T2 or T3) of the intervention was determined using paired t-test for parametric values and Wilcoxon test for nonparametric values.
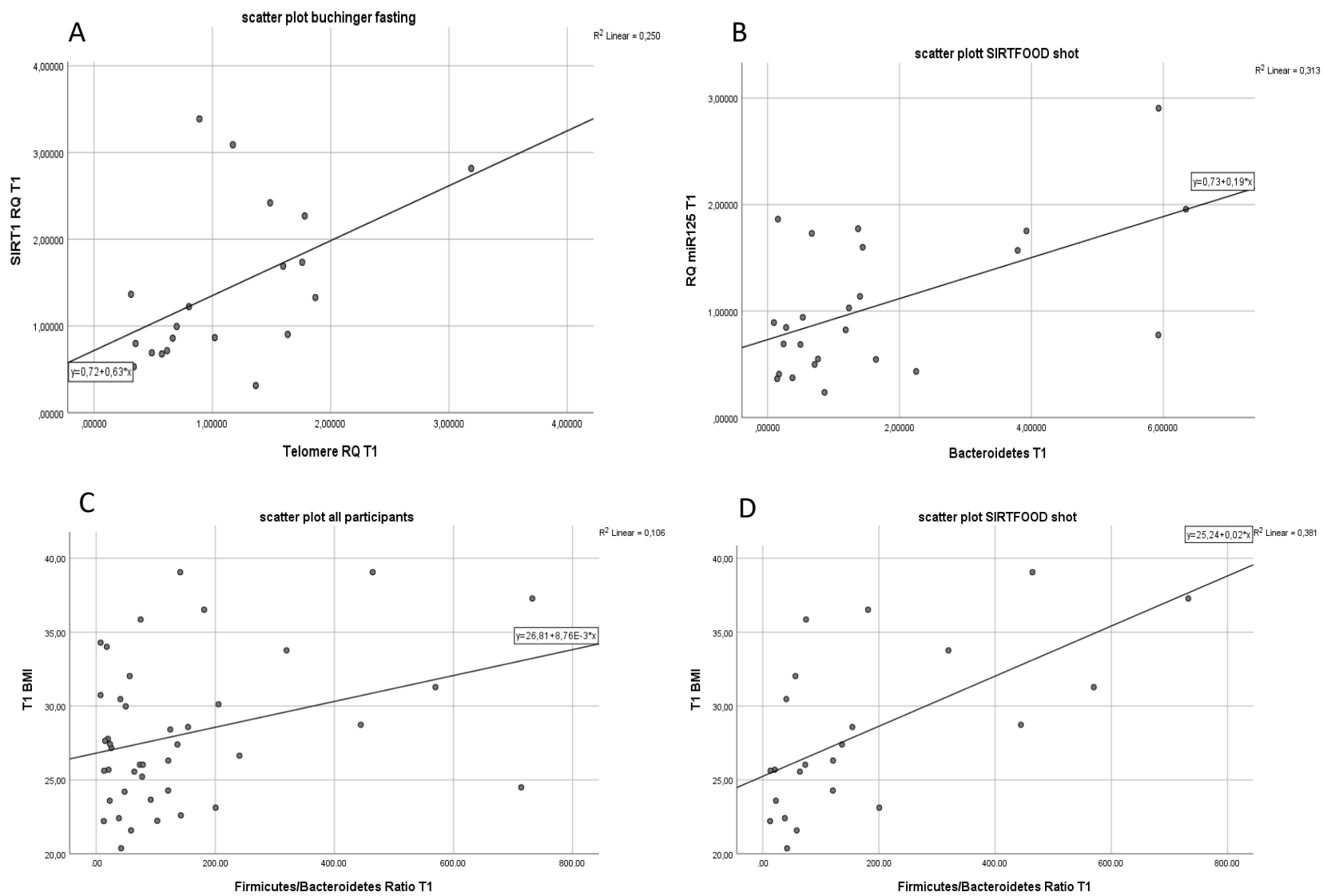

Figure 8: spss output scatter plots. (A) shows a positive correlation between telomere length and SIRT1 expression for buchinger fasting at baseline. Bacteroidetes and miR125b-5p positively correlated in the SIRTFOOD shot intervention at baseline(B). For all participants the ratio of Firmicutes/Bacteroidetes increased with higher BMI (C), which was also seen for the SIRTFOOD shot intervention Discussion (D). Statistical significance was defined as $p<0.05$. 


\section{DISCUSSION}

CR has been widely discussed for its beneficial effect on human health. In animal studies, CR was reported to elevate sirtuin expression but to our knowledge, the effect of sirtuin expression after consecutive fasting days has not yet been studied in humans. The term fasting mimicking diet has been increasingly used in literature recently and its positive impact on human health has already been revealed[5]. Anyway, many fasting mimicking diets, like the SIRTFOOD diet developed by Goggins and Matten, result in a reduction of caloric intake, when changing their dietary habits[29,5]. Our study was conducted upon our vitro study with different secondary plant ingredients regarding senescence and sirtuin expression. Now we wanted to observe the potential of periodic fasting and/or supplementing of a combination of secondary pant ingredients without changing dietary habits on sirtuin expression. Certain polyphenol combinations exhibit synergistic effects with a higher biological activity than the sum of the individual ones[30,31]. Various aging biomarkers have been identified to monitor the personal response on lifestyle, nutrition and environment. Among them, LINE1 methylation as a marker for global methylation level has been positively associated with $\mathrm{BMI}$ and obesity ${ }^{32}$. Our results showed no differences in LINE1 methylation before and after the intervention, like in the study of Duggen et al.[33]. However, as Marques-Rocha et al. could observe, the methylation level of participants in our study also correlated with body weight[34,33]. Yet, they found a higher methylation level in individuals with lower body fat mass, which we could not see in our data[34]. Regarding telomere length, we observed a positive correlation with weight loss in contrast to Mason et al., who did not see any changes resulting from different diet and sport interventions. One possible explanation might be the differences in study populations[35]. Our study constitutes a very heterogenous group of individuals, to assess potential wide-range effects. Furthermore, we observed a correlation between LINE1 hypomethylation and increased telomere length, assuming LINE1 methylation decreases with lower body fat mass thus resulting in higher telomere length. Baseline telomere length attenuated with age in the SIRTFOOD shot group, but not for the buchinger fasting. This result may be explained by the population size, intervention time and age distribution of the different interventions. Telomere shortening is a hallmark of aging which implicates driving pathologies and aging[36,1]. Both interventions increased SIRT1 significantly. Glucose deprivation, like in fasting leads to a switch in AMP/ATP and increased $\mathrm{NAD}^{+}$ concentrations, subsequently activating AMPK and sirtuins. Bioactive substances target sirtuins, by targeting nicotinamide phosphoribosyltransferase (NAMPT), an enzyme in the NAD salvage pathway, which leads to an increased $\mathrm{NAD+} / \mathrm{NADH}$ ratio. Polyphenols can target AMPK, by blocking the FOF1ATPase/ATP synthase, the complex IV in the respiratory chain reaction in mitochondria, leading to a switch in the AMP/ATP ratio and elevation on NAD+ in the cell[37,38]. At baseline, telomere length positively correlated with SIRT1 expression in the fasting intervention and with SIRT6 for SIRTFOOD shot. A study conducted by Palacious et al. demonstrates SIRT1 having a positive effect on telomere length, but only in mouse models[39]. SIRT6 was found to protect DNA by maintaining telomeres and modulating 5-hydroxymethylcytosine, the first oxidative product in the demethylation of 5methycytosine[12]. Moreover, SIRT6 depletion results in abnormal telomeres, leading to genomic abnormalities and premature senescence[40].Although we observed SIRT1 expression increased significantly in both groups, no changes in telomere length were seen before and after the interventions. Another longevity-related 
protein of the sirtuin family is SIRT3, which attenuates ROS through superoxide dismutase 2 (SOD2) delays cell senescence and decreases oxidative[12,41]. SIRT3, a mitochondrial sirtuin that regulates the enzyme 3-hydroxy-3-methylglutaryl-CoA synthase, impacts ketone body production[42]. Although SIRT3 activation was not significantly, periodic fasting elevated BHB to an extend of $5.7 \mathrm{mM}$. However, ketosis and ketone body production were very unequal because participants were allowed to consume a spoon of honey in case of dizziness, which could have an impact on ketone body generation. Ketogenesis is a metabolic mechanism in mitochondria responding to fasting ${ }^{43}$. Increased PDK4 gene expression is usually observed during fasting, which inhibits pyruvate dehydrogenase complex, conversion of pyruvate to acetyl CoA decreases and at the same time $ß$-oxidation increases to provide energy[8]. Remarkably, we could see this for the SIRTFOOD shot group, assuming increased fatty acid oxidation also in the supplementation group $[44,45,46]$. It is well known, that an increased ß-oxidation and decreased glucose concentrations lead to FoxO1 activation[46]. The axis between SIRT1/ FoxO1 and PDK4 is not yet fully understood, but SIRT1 modulates upstream signaling of FoxO1 and its activity improves glucose utilization[44]. Many miRNAs have been studied and new connections with our lifestyle and pathways can be generated, thus pattern can be seen of miRNAs and age-related diseases, like lower miRlet $7 b-5 p$ levels in cardiovascular diseases and diabetes type 2[47]. Although our results in this regard were not significant, Cannataro et al. showed that miRlet $7 b-5 p$ increased during ketogenic diet[17]. Overexpression of miR21-5p, which our results show for both interventions, is often associated with inflammation and cancerogenesis, but can also dampen cytokine secretion mediated by toll-like receptor 4 (TLR4)[48,49]. Several papers implicated the interaction of SIRT1 downregulation via miR34a-5p overexpression[50,51]. miR34a is not only associated with different cancer types, but moreover it also interacts with the cardiovascular system and metabolism by modulating SIRT1 expression. Overexpression of miR34a inhibits SIRT1 expression and endothelial senescence. miR34a is elevated in diet-induced obesity with a concomitant decrease in insulin sensitivity[16]. At the baseline for the SIRTFOOD shot group, we could also observe that an overexpression of miR34a-5p correlates with decreased SIRT1 expression. However, SIRT1 can also be expressed in the gut which plays an important role in health and disease[52]. Gene expression is modulated by lifestyle, furthermore, miRNAs interact with our microbiome and their metabolites. Nonetheless, the microbiome can be also modulated with nutrition. Similarly like recently observed in another survey, we assessed a positive correlation of Firmicutes /Bacteroidetes ratio and BMI, whereas the ratio decreased after both intervention[53]. Furthermore, in the SIRTFOOD shot group a significant increase of Actinobacteria could be seen, which agrees with the literature, where secondary plant ingredients, like gallic acid and resveratrol were observed to modulate the abundance of Bifidobacteria, which constitute a major part of the phylum Actinobacteria in the human gut[19,52]. However, the prebiotic agent, which the supplement consists of, could be a more reasonable explanation for this change[54]. Nevertheless, Bifidobacteria exhibit beneficial effects on the host as it promotes gut maturation and integrity, protects against pathogens, and modulates immunity. Moreover, agerelated changes in physiology and function of the gastrointestinal tract could result in a decline in Bifidobacteria, which could be reversed with the supplement[55]. We found only two other studies assessing changes in gut diversity after buchinger fasting. Like Mesnage et al., we observed a major 
decrease in Firmicutes and a significant increase in Proteobacteria after periodic fasting. Proteobacteria are usually associated with inflammatory bowel disease, but it is important to mention, that these are strain dependent[18]. Differences in miRNAs expression patterns can be seen in stool and plasma samples regarding nutritional habits[56]. Hewel et al., could identify that miR125b and Bacteroidetes have multiple potential identical targets, for which we saw a significant positive interaction with the supplement[57,58]. Moreover, Martinez et al. showed that miR125b-5p and miR16-5p are downregulated in participants with irritated bowel syndrome, assuming that the increased amount of Bacteroidetes and overexpression of miR125b-5p after SIRTFOOD shot supplementation could have a positive impact on intestinal barrier function[59]. This interaction of course could not be seen for the fasting intervention because without solid food for 5 days this bacterial phylum cannot grow and therefore, no changes in the amount of Bacteroidetes were observed for the fasting intervention. Nevertheless, for this group we observed a positive correlation of Tenericutes producing butyrate, which was already identified as a butyrate producer by Vital et al.[60]. The anti-inflammatory properties of butyrate have many beneficial effects on health[61]. At last, we identified a positive correlation of the abundance of Bacteroidetes, another butyrate producer, with telomere length and a decrease of Verrucomicrobia with age, which further affects SIRT6 expression for this group $(p<0.05)$. Fransen et al. observed the same result for mice and the amount of this phylum decreases with age, but to our knowledge, little is published about sirtuins stimulating humans microbiota[62].

\section{Limitations:}

Like in various other in vivo studies, the bioavailability of the bioactive substances is a major concern. Testing the compound individually would be interesting to investigate. The effects of the different microbial compositions are very strain-specific, subsequently, changes in bacterial strains and its interaction are of high interest for our future studies. The effects of polyphenols and the galactooligosaccharides in the drink should be elucidated specifically.

Conclusion and perspectives: Our study provides results from 5 days of periodic fasting and a fasting mimetic supplement within gene expression involved in health span and longevity. Periodic fasting activated SIRT1 and modulated gut microbiota. Supplementing a combination of bioactive compounds without reducing caloric intake shows similar expression patterns of our selected mRNA and miRNAs. Changes in the microbiota, especially Actinobacteria, a butyrate producing bacteria phylum were observed with the supplement. Both interventions revealed results with beneficial outcomes for human health and confirm the effects of fasting on longevity associated mechanisms but also suggest that an intervention with a combination of certain sirtuin activating bioactive compounds addresses some of these effects.

List of Abbreviations: Body mass index (BMI), reactive oxygen species (ROS), calorie restriction (CR), pyruvate dehydrogenase kinase isoform 4 (PDK4), adenosine monophosphate (AMP), AMP-activated protein kinase (AMPK), adenosine triphosphate (ATP), silent information regulator 2 (SIR2), nicotinamide adenine dinucleotide (NAD+), superoxide dismutase 2 (SOD2), the Forkhead box subgroup O (FoxO), MutL homolog 1 (MLH1), $\beta$-hydroxybutyrate (BHB), Long Interspersed Nuclear Elements (LINE), microRNAs (miRNAs), timepoint 1 (T1), timepoint 2 (T2), timepoint 3 (T3), short chain fatty acids (SCFAs), , medium chain fatty acid (MSCFa), tricarboxylic acid (TCA), toll-like receptor 4 (TRL4) 
Author Contributions: S.L., A.H designed the research. S.L., H.B., I.H., conducted the research. S.L., H.B, K.D., T.D. and J.K. L.S. and B.H. performed clinical analysis. S.L., H.B., performed statistical analyses. S.L., A.P and A.H. wrote the manuscript. A.H. had primary responsibility for the final content. All authors read and approved the final version of the manuscript.

Declaration of competing interest: The authors declare that they have no known competing financial interests or personal relationships that could have appeared to influence their work in this paper.

Acknowledgements and Funding: We thank the members of University of Vienna; Department for Nutritional Science, Biomes NGS GmbH and the Monastory Pernegg for their assistance and support with the trial. The study was funded by grands of Austrian research funding agency

\section{REFERENCES:}

1. G. Lopez-Otin, C., Blasco, MA., Partridge, L., Serrano, M. and Kroemer, "Hallmark of aging," Cell 2013, vol. 153, no. 6. pp. 1194-1217.

2. A. Guillaumet-Adkins, Y. Yañez, M. D. Peris-Diaz, I. Calabria, C. Palanca-Ballester, and J. Sandoval, "Epigenetics and Oxidative Stress in Aging," Oxid. Med. Cell. Longev. 2017, doi: 10.1155/2017/9175806.

3. S. Ahadi, W. Zhou, S. Schüssler-Fiorenza Rose, M. Sailani, K. Contrepois, M. Avina, M. Ashland et al., "Personal aging markers and ageotypes revealed by deep longitudinal profiling," Nat. Med. 2020, doi: 10.1038/s41591-019-07195.

4. C. W. Bales and W. E. Kraus, "Caloric restriction: Implications for human cardiometabolic health," J. Cardiopulm. Rehabil. Prev. 2013, doi:10.1097/HCR.0b013e318295019e.

5. M. Wie, S. Brandhorst, M. Shelehchi, H. Mirzaei, C. W. Cheng, J. Budniak, S. Groshen et al., "HHS Public Access," Sci Transl Med. 2017 February 15; 9(377): . doi:10.1126/scitranslmed.aai8700

6. D. N. Gross, A. P. J. Van Den Heuvel, and M. J. Birnbaum, "The role of FoxO in the regulation of metabolism," Oncogene 2008, vol. 27, no. 16, pp. 2320-2336, doi: 10.1038/onc.2008.25.

7. J. C. Newman and E. Verdin, " $\beta$-hydroxybutyrate: Much more than a metabolite," Diabetes Research and Clinical Practice. 2014, doi: 10.1016/j.diabres.2014.08.009.

8. M. Bouwens, L. A. Afman, and M. Müller, "Fasting induces changes in peripheral blood mononuclear cell gene expression profiles related to increases in fatty acid $\beta$ oxidation: Functional role of peroxisome proliferator- activated receptor $\alpha$ in human peripheral blood mononuclear cells," Am. J. Clin. Nutr. 2007, vol. 86, no. 5, pp. 1515-1523, 2007, doi: 10.1093/ajcn/86.5.1515.

9. S. Martin, T. Hardy, and T. Tollefsbol, "Medicinal Chemistry of the Epigenetic Diet and Caloric Restriction," Curr. Med. Chem., 2013, doi: 10.2174/09298673113209990189.

10. C. Cantó and J. Auwerx, "Calorie restriction: Is AMPK a key sensor and effector?," Physiology Aug. 2011, vol. 26, no. 4. pp. 214-224, doi: 10.1152/physiol.00010.2011.

11. B. Shoba, Z. M. Lwin, L. S. Ling, B. H. Bay, G. W. Yip, and S. D. Kumar, "Function of sirtuins in biological tissues," Anat. Rec. 2009, vol. 292, no. 4, pp. 536-543, doi: 10.1002/ar.20875

12. Y. Wang, J. He, M. Liao, M. Hu, W. Li, H. Ouyang, X. Wang et al., "An overview of Sirtuins as potential therapeutic target: Structure, function and modulators," Eur. J. Med. Chem. 2019, vol. 161, pp. 48-77, doi: 10.1016/j.ejmech.2018.10.028.

13. D. H. Kim, M. H. Park, E. K. Lee, Y. J. Choi, K. W. Chung, K. M. Moon, M. J. Kim, et al., "The roles of FoxOs in modulation of aging by calorie restriction," Biogerontology 2014 , vol. 16, no. 1, doi: 10.1007/s10522-014-9519-y.

14. K. Makwana, S. A. Patel, N. Velingkaar, J. S. Ebron, G. C. Shukla, and R. V. Kondratov, "Aging and calorie restriction regulate the expression of miR-125a-5p and its target genes Stat3 Casp2 and Stard13," Aging (Albany. NY). 2017, vol. 9, no. 7, pp. 1825-1843, doi: 10.18632/aging.101270.

15. M. Yamakuchi, "MicroRNA regulation of SIRT1," Front. Physiol. 2012, vol. 3 MAR, no. March, pp. 1-8, doi: 10.3389/fphys.2012.00068.

16. R. Cannataro, M. Perri, L. Gallelli, M. C. Caroleo, G. De Sarro, and E. Cione, "Ketogenic Diet Acts on Body Remodeling and MicroRNAs Expression Profile," MicroRNA 2018, vol. 8, no. 2, pp. 116-126, doi: $10.2174 / 2211536608666181126093903$.

17. R. Mesnage, F. Grundler, A. Schwiertz, Y. Le Maho, and F. Wilhelmi de Toledo, "Changes in human gut microbiota composition are linked to the energy metabolic switch during $10 \mathrm{~d}$ of Buchinger fasting," J. Nutr. Sci. 2019, vol. 8, p. e36, doi: 10.1017/jns.2019.33.

18. M. A. Farag, A. Abdelwareth, I. E. Sallam, M. el Shorbagi, N. Jehmlich, K. Fritz-Wallace, S. S. Schäpe et al., "Metabolomics reveals impact of seven functional foods on metabolic pathways in a gut microbiota model," Journal of Advanced Research 2020, vol. 23. pp. 47-59, 2020, doi: 10.1016/j.jare.2020.01.001.

19. M. Colitti and S. Grasso, "Nutraceuticals and regulation of adipocyte life: Premises or promises," BioFactors. 2014, doi: 10.1002/biof.1164

20. F. Gurău, S. Baldoni, F. Prattichizzo, E. Espinosa, F. Amenta, A. D. Procopio, M. C. Albertini et al., "Anti-senescence compounds : A potential nutraceutical approach to healthy aging," Elsevier 2018, vol. 46, no. April, pp. 14-31, doi: 10.1016/j.arr.2018.05.001.

21. F. Pietrocola, J. Pol, E. Vacchelli, S. Rao, D. P. Enot, E. E. Baracco, S. Levesque, "Caloric Restriction Mimetics Enhance Anticancer Immunosurveillance" HHS Public Access, Cancer Cell. 2016 July 11; 30(1): 147-160. doi:10.1016/j.ccell.2016.05.016.

22. M. Malavolta, M. Bracci, L. Santarelli, M. A. Sayeed, E. Pierpaoli, R. Giacconi, L. Costarelli et al., "Inducers of senescence, toxic compounds, and senolytics: The multiple faces of Nrf2-activating phytochemicals in cancer adjuvant therapy," Mediators Inflamm. 2018, vol. 2018, doi: 10.1155/2018/4159013.

23. B. Lindström and M. Eriksson, "Salutogenesis," Journal of Epidemiology and Community Health 2005,, vol. 59, no. 6. J Epidemiol Community Health, pp. 440-442, doi: 10.1136/jech.2005.034777. 
24. M. Remely, B. Hippe, I. Geretschlaeger, S. Stegmayer, I. Hoefinger, and A. Haslberger, "Increased gut microbiota diversity and abundance of Faecalibacterium prausnitzii and Akkermansia after fasting: A pilot study," Wien. Klin. Wochenschr., 2015, doi: 10.1007/s00508-015-0755-1.

25. Xi. Duan, Y. Yang, S. Wang, X. Feng, T. Wang, P. Wang, S. Liu et al., "Changes in the expression of genes involved in cell cycle regulation and the relative telomere length in the process of canceration induced by omethoate," Tumor Biol. 2017, vol. 39, no. 7, pp. 1-10, doi: $10.1177 / 1010428317719782$.

26. R. Peters, J. Hellenbrand, Y. Mengerink, and S. Van Der Wal, "On-line determination of carboxylic acids, aldehydes and ketones by high-performance liquid chromatographydiode array detection-atmospheric pressure chemical ionisation mass spectrometry after derivatization with 2 nitrophenylhydrazine," J. Chromatogr. A 2004, vol. 1031 no. 1-2, pp. 35-50, doi: 10.1016/j.chroma.2003.10.100.

27. A. Goggins and G. Mattens "The Sirtfood Diet." first published in Great Britain in 2016 by yellow kite, http://www.thesirtfooddiet.com.

28. S. Lilja, J. Oldenburg, A. Pointner, L. Dewald, M. Lerch, B. Hippe, O. Switzeny et al., "Epigallocatechin Gallate Effectively Affects Senescence and Anti- SASP via SIRT3 in 3T3-L1 Preadipocytes in Comparison with Other Bioactive Substances," 2020, doi.org/10.1155/2020/4793125

29. D. Skroza, I. Generalić Mekinić, S. Svilović, V. Šimat, and V. Katalinić, "Investigation of the potential synergistic effect of resveratrol with other phenolic compounds: A case of binary phenolic mixtures," J. Food Compos. Anal. 2015, vol. 38, pp. 13-18, 2015, doi: 10.1016/j.jfca.2014.06.013.

30. L. Zhang, D. J. McClements, Z. Wei, G. Wang, X. Liu, and F. Liu, "Delivery of synergistic polyphenol combinations using biopolymer-based systems: Advances in physicochemical properties, stability and bioavailability," Crit. Rev. Food Sci. Nutr. 2020, vol. 60, no. 12, pp. 2083-2097, doi: 10.1080/10408398.2019.1630358.

31. M. Garcia-Lacarte, F. I. Milagro, M. A. Zulet, J. A. Martinez, and M. L. Mansego, "LINE-1 methylation levels, a biomarker of weight loss in obese subjects, are influenced by dietary antioxidant capacity," Redox Rep. 2016, vol. 21, no. 2, pp. 67-74, doi: 10.1179/1351000215Y.0000000029.

32. C. Duggan, L. Xiao, M. B. Terry, and A. McTiernan, "No effect of weight loss on LINE-1 methylation levels in peripheral blood leukocytes from postmenopausal overweight women," Obesity 2014, vol. 22, no. 9, pp. 2091-2096, doi: 10.1002/oby.20806.

33. J. L. Marques-Rocha, F. I. Milagro, M. L. Mansego, D. M. Mourão, J. A. Martínez, and J. Bressan, "LINE-1 methylation is positively associated with healthier lifestyle but inversely related to body fat mass in healthy young individuals," Epigenetics 2016, vol. 11, no. 1, pp. 49-60, 2016, doi: 10.1080/15592294.2015.1135286.

34. C. Mason, R. Risques, L. Xiao, C. R. Duggan, I. Imayama, K. L. Campbell, A. Kong et al., "Independent and combined effects of dietary weight loss and exercise on leukocyte telomere length in postmenopausal women," Obesity 2013, vol. 21 , no. 12 , pp. 1-15, 2013, doi: 10.1002/oby. 20509.

35. M. A. Shammas, "Telomeres, lifestyle, cancer, and aging Masood", NIH public Access Cur. Opin. Clin. Nutr. Metab. Care 2011, 14(1): 28-34. doi:10.1097/MCO.0b013e32834121b1

36. W. Grabowska, E. Sikora, and A. Bielak-Zmijewska, "Sirtuins, a promising target in slowing down the ageing process," Biogerontology 2017, vol. 18, no. 4, pp. 447-476, doi: 10.1007/s10522-017-9685-9.

37. J. Zheng and V. D. Ramirez, "Inhibition of mitochondrial proton FOF1-ATPase / ATP synthase by polyphenolic phytochemicals," British Journal of Pharmacology 2000,
1115 - 1123.

38. J. A. Palacios, D. Herranz, M. L. De Bonis, S. Velasco, M. Serrano, and M. A. Blasco, "SIRT1 contributes to telomere maintenance and augments global homologous recombination," J. Cell Biol. 2010, vol. 191, no. 7, pp. 12991313, doi: 10.1083/jcb.201005160.

39. R. I. Tennen, D. J. Bua, W. E. Wright, and K. F. Chua, "SIRT6 is required for maintenance of telomere position effect in human cells", HHS Public Access, Nat Commun. 2012 ; 2: 433. doi:10.1038/ncomms1443.

40. B. Kincaid and E. Bossy-Wetzel, "Forever young: SIRT3 a shield against mitochondrial meltdown, aging, and neurodegeneration," Front. Aging Neurosci. 2013, vol. 5, no. SEP, pp. 1-13, doi: 10.3389/fnagi.2013.00048.

41. T. Shimazu, M. D. Hirschey, L. H., K. E. Dittenhafer-Reed, B. Schwer, D. B. Lombard, Y. Li et al., "SIRT3 deacetylates mitochondrial 3-hydroxy-3-methylglutaryl CoA synthase 2 and regulates ketone body production," Cell Metab. Dec. 2010, vol. 12, no. 6, pp. 654-661, doi: 10.1016/j.cmet.2010.11.003.

42. Y. Zhu, Y. Yan, D. R. Gius, and A. Vassilopoulos, " Metabolic regulation of sirtuins upon fasting and the implication for cancer" HHS Public Access, Curr Opin Oncol. 2013 November ; 25(6): 630-636. doi:10.1097/01.cco.0000432527.49984.a3.

43. L. L. Spriet, R. J. Tunstall, M. J. Watt, K. A. Mehan, M. Hargreaves, and D. Cameron-Smith, "Pyruvate dehydrogenase activation and kinase expression in human skeletal muscle during fasting," J. Appl. Physiol. 2004,, vol. 96, no. 6, pp. 2082-2087, doi: 10.1152/japplphysiol.01318.2003.

44. T. K. Sin, B. Y. Yung, and P. M. Siu, "Modulation of SIRT1foxo1 signaling axis by resveratrol: Implications in skeletal muscle aging and insulin resistance," Cell. Physiol. Biochem. 2015, vol. 35, no. 2, pp. 541-552, doi: 10.1159/000369718.

45. S. Zhang, M. W. Hulver, R. P. McMillan, M. A. Cline, and E. R. Gilbert, "The pivotal role of pyruvate dehydrogenase kinases in metabolic flexibility," Nutr. Metab. 2014, vol. 11, no. 1, pp. 1-9, doi: 10.1186/1743-7075-11-10.

46. Y. Huang, Y. Yan, W. Xv, G. Qian, C. Li, H. Zou and Y. Li., "A New Insight into the Roles of MiRNAs in Metabolic Syndrome," Biomed Res. Int. 2018, vol. 2018, doi: $10.1155 / 2018 / 7372636$

47. H. Go, J. Jang1, P.-J. Kim, Y.-G. Kim, S. J. Nam, J. H. Paik, T. M. Kim et al., "MicroRNA-21 plays an oncogenic role by targeting FOXO1 and activating the PI3K/AKT pathway in diffuse large B-cell lymphoma," Oncotarget 2015, vol. 6, no. 17, pp. 15035-15049, doi: 10.18632/oncotarget.3729.

48. K. K. Kaur, G. N. Allahbadia and M. Singh, "An Update on Microrna's and Metabolic Regulation with Future Therapeutic Potentials Regarding Diagnosis and Treatment of Obesity, Metabolic Syndrome and Other Related Disorders," J. Heal. Med. Informatics 2015, vol. 06, no. 02, doi: 10.4172/2157-7420.1000184

49. M. Yamakuchi and C. J. Lowenstein, "MiR-34, SIRT1 and p53: The feedback loop," Cell Cycle 2009, vol. 8, no. 5, pp. 712-715, doi: 10.4161/cc.8.5.7753.

50. M. Yamakuchi, M. Ferlito, and C. J. Lowenstein, “ miR-34a repression of SIRT1 regulates apoptosis" PNAS 2008105 (36) 1342113426; doi.org/10.1073/pnas.080161 $\underline{3105}$

51. S. E. Lakhan and A. Kirchgessner, "Gut microbiota and sirtuins in obesity-related inflammation and bowel dysfunction," Journal of Translational Medicine. 2011, doi: 10.1186/1479-5876-9-202.

52. A. Koliada, G. Syzenko, V. Moseiko, L. Budovska, K. Puchkov, V. Perederiy, Y. Gavalko et al., "Association between body mass index and Firmicutes/Bacteroidetes ratio in an adult Ukrainian population," BMC Microbiol., 
BMC Microbiology (2017) 17:120 DOI 10.1186/s12866017-1027-1

53. E. E. Canfora, C. M. van der Beek, G. D.A. Hermes, G. H. Goossens, J. W.E. Jocken, J. J. Holst, H. M. van Eijk et al., "Supplementation of Diet With Galacto-oligosaccharides Increases Bifidobacteria, but Not Insulin Sensitivity, in Obese Prediabetic Individuals," Gastroenterology 2017, vol. 153, no. 1 , pp. 87-97.e3, doi: 10.1053/j.gastro.2017.03.051.

54. R. Nagpala, R. Mainalia, S. Ahmadia, S. Wanga, R. Singha, K. Kavanaghc, D. W. Kitzmand et al., "Gut microbiome and aging: Physiological and mechanistic insights," Nutr. Heal. Aging 2018, vol. 4, no. 4, pp. 267-285, doi: 10.3233/NHA170030.

55. S. Tarallo, B. Pardini, G. Mancuso, F. Rosa, C. Di Gaetano, F. Rosina, P. Vineis et al., "MicroRNA expression in relation to different dietary habits: A comparison in stool and plasma samples," Mutagenesis, 2014, doi: 10.1093/mutage/geu028.

56. J. Vulevic, A. Drakoularakou, P. Yaqoob, G. Tzortzis, and G. R. Gibson, "Modulation of the fecal microflora profile and immune function by a novel trans-galactooligosaccharide mixture (B-GOS) in healthy elderly volunteers," $\mathrm{Am}$. J. Clin. Nutr. 2008, vol. 88, no. 5, pp. 1438-1446, doi: 10.3945/ajcn.2008.26242.

57. C. Hewel, J. Kaiser, A. Wierczeiko, J. Linke, C. Reinhardt, K. Endres and S. Gerber, "Common miRNA Patterns of
Alzheimer's Disease and Parkinson's Disease and Their Putative Impact on Commensal Gut Microbiota," Front Neurosci. 2019, vol. 13, no. March, pp. 1-13, doi: 10.3389/fnins.2019.00113.

58. C. Martínez, B. K. Rodiño-Janeiro, B. Lobo, M. L. Stanifer, B. Klaus, M. Granzow, A. M. González-Castro et al., "MiR-16 and miR-125b are involved in barrier function dysregulation through the modulation of claudin-2 and cingulin expression in the jejunum in IBS with diarrhoea," Gut 2017, vol. 66, no. 9, pp. 1597-1610, doi: 10.1136/gutjnl-2016-311477.

59. M. Vital, A. C. Howe, and J. M. Tiedje, "Revealing the bacterial butyrate synthesis pathways by analyzing (meta)genomic data," MBio 2014, vol. 5, no. 2, pp. 1-11, doi: $10.1128 / \mathrm{mBio} .00889-14$

60. R. B. Canani, M. Di Costanzo, L. Leone, M. Pedata, R. Meli, and A. Calignano, "Potential beneficial effects of butyrate in intestinal and extraintestinal diseases," World $J$ Gastroenterol. 2011, vol. 17, no. 12, pp. 1519-1528, doi: 10.3748/wjg.v17.i12.1519.

61. F. Fransen, A. A. van Beek, T. Borghuis, S. El Aidy, F. Hugenholtz, C. van der Gaast - de Jongh, H. F. J. Savelkoul et al., "Aged gut microbiota contributes to systemical inflammaging after transfer to germ-free mice," Front. Immunol. 2017, vol. 8, no. NOV, pp. 1-12, doi: 10.3389/fimmu.2017.01385. 